\title{
HPV vaccination in Japan: results of a 3-year follow-up survey of obstetricians and gynecologists regarding their opinions toward the vaccine
}

\author{
Masaaki Sawada $^{1}$ - Yutaka Ueda ${ }^{1}\left(\right.$ Asami Yagi $^{1} \cdot$ Akiko Morimoto $^{1} \cdot$ Ruriko Nakae $^{1} \cdot$ Reisa Kakubari $^{1}$. \\ Hazuki Abe $^{1}$ ' Tomomi Egawa-Takata ${ }^{1}$ Tadashi Iwamiya $^{1} \cdot$ Shinya Matsuzaki $^{1} \cdot$ Eiji Kobayashi $^{1}$. \\ Kiyoshi Yoshino $^{1} \cdot$ Tadashi Kimura $^{1}$
}

Received: 8 May 2017 / Accepted: 23 August 2017 / Published online: 6 October 2017

(C) The Author(s) 2018. This article is an open access publication

\begin{abstract}
Background In Japan, the cervical cancer preventative HPV vaccination rate has dramatically declined, directly as a result of repeated broadcasts of so-called adverse events and the resulting suspension of the government's recommendation. Our previous survey of obstetricians and gynecologists in Japan regarding their opinions toward HPV vaccination revealed that these key specialists were as negatively influenced by the reports of purported negative events as were the general population. Here, we report a 3-year follow-up survey of these clinicians.

Methods We reused the same questionnaire format as used in our 2014 survey, but added new questions concerning opinions regarding a WHO statement and reports of a Japanese nation-wide epidemiological study related to the adverse events, released in 2015 and 2016, respectively.

Results The response rate was 46\% (259/567): 5 (16.1\%) of 31 doctors had inoculated their own teenaged daughters during the time period since the previous survey, despite the continued suspension of the governmental recommendation, whereas in the previous survey none of the doctors had done so. Among the respondents, the majority claimed awareness of the recent pro-vaccine WHO statement (66.5\%), and of the report of a Japanese epidemiological study (71.5\%), and a majority affirmed they currently held positive opinions of the safety $(72.7 \%)$ and effectiveness $(84.3 \%)$ of the HPV vaccine.
\end{abstract}

Yutaka Ueda

ZVF03563@nifty.ne.jp

1 Department of Obstetrics and Gynecology, Osaka University Graduate School of Medicine, 2-2, Yamadaoka, Suita, Osaka 565-0871, Japan
Conclusions Our re-survey of Japan's obstetricians and gynecologists regarding their opinions about the HPV vaccine found that their opinions have changed, potentially leading to a more positive future re-engagement for HPV vaccination in Japan.

Keywords HPV vaccine · Adverse event · Suspension . Obstetrics · Gynecology · Opinion
Abbreviations
HPV Human papillomavirus
MHLW The Ministry of Health, Labour and Welfare
WHO World Health Organization

\section{Introduction}

In Japan, the incidence of cervical cancer has worrisomely begun to increase recently, especially in younger generations [1]. To combat the scourge of human papillomavirus (HPV)-caused cervical cancer, the HPV vaccine was first approved for use clinically in 2009 and was followed by an urgent promotional campaign for vaccination conducted by the Ministry of Health, Labour and Welfare (MHLW) in 2010. This campaign was a huge success, and the HPV vaccination status approached $70-80 \%$ of the targeted age group of young girls [2-4] until 2012. Then, in April 2013, buoyed by their early success, MHLW initiated periodic vaccinations, targeting girls 12-16 years of age.

Unfortunately, as the mass vaccinations began in the early spring of 2013, media reports of rare but serious adverse events alleged to have occurred in the young girls after their HPV vaccination began to circulate, soon creating a strong state of public distrust. Consequently, the MHLW responded to mounting media pressure by announcing a suspension of 
their blanket recommendation for the HPV vaccine in June 2013. That suspension caused a complete and utter collapse of HPV vaccination in Japan until the present time [2-4].

Our previous studies showed that acceptance of the HPV vaccine was determined predominantly by a young girl's mother's perceptions of the risks versus the benefits of the HPV vaccine, rather than by the daughter's perceptions. We found that the mothers' perceptions greatly overestimated the frequency of the adverse events that had been reported by media, resulting in their hesitation to urge their daughters to be inoculated [5].

Recent publications have revealed new information about the implementation of HPV vaccines. A trusted medical provider's recommendation of the vaccine was found to be key to any approach, as was routinely offering the vaccine at the same time as other universally recommended adolescent immunizations [6].

In August 2014, 14 months since the suspension of the governmental recommendation of the HPV vaccine, we conducted our first survey on the opinions about the HPV vaccine of our obstetricians and gynecologists [7], because they are the first-line medical providers most likely to encourage HPV vaccine to mothers. Our study revealed that, as in the general population, obstetricians and gynecologists were significantly negatively influenced by both the media reports of the alleged adverse events of the HPV vaccine and the suspension of the government's recommendation of the vaccine.

In 2015, WHO clearly stated that "policy decisions based on weak evidence, leading to lack of use of safe and effective vaccines, can result in real harm," referring directly to the worrisome circumstances in Japan [8]. Meanwhile, a nationwide epidemiological study of adolescents in Japan, of the diverse adverse symptoms alleged to have been caused by the HPV vaccine including pain and motor disability, was conducted in 2015 and 2016 by Professor Sobue et al. under MHLW financial support. According to the Sobue Report, even without HPV vaccination, the girls in the targeted age group suffered from similar symptoms as purported to have occurred following HPV vaccination [9].

Now approaching 4 years, the suspension of the governmental recommendation of the HPV vaccine has continued. Here, following WHO's pro-vaccine statement and the Sobue Report, we have conducted a second round of surveys of Japanese obstetricians and gynecologists to determine the change in their opinions toward the HPV vaccine.

\section{Methods}

We posted our questionnaire on HPV vaccine in January 2017 to 567 doctors who had previously trained in the Department of Obstetrics and Gynecology of the Osaka University Hospital or one of its associated hospitals and clinics. The same structured format questionnaire was used, with closed-ended questions, including the original questions about their personal opinions about HPV vaccination, cervical cancer screening, and their own daughters' HPV vaccination status. We added new questions to evaluate their awareness about the WHO statement [8] and the Sobue Report [9], and their opinion toward them. We compared the doctors' intentions to recommend HPV vaccination to teenagers in their care, their thoughts regarding whether the government should restart its recommendation for the vaccine, and the number of the physicians who had inoculated their teenaged daughters since the first survey, to the survey results from 2014.

\section{Statistics}

Fisher's exact test was used for statistical analysis; the level of statistical significance was set at $p=0.05$.

\section{Results}

\section{Characteristics of the responders to the present survey}

Of the 567 doctors to whom the survey was sent, 259 replied by February 28, 2017, a response rate of $46 \%$, which was similar to that of the previous survey (46\%) [7]. The relevant characteristics of the responding doctors are listed in Table 1 . Of the responders, $80.8 \%$ had at least one daughter who was under 30 years old at the taking of the survey, which was significantly higher than that in the previous survey $(p<0.01)$. There was no statistically significant difference in the other characteristics (data not shown). Among the responders, $98.1 \%$ had conducted medical examination of at least one patient with cervical cancer, and $87.9 \%$ had personally experienced the death of the patient with cervical cancer.

\section{Comparison of baseline opinions toward the HPV vaccine between the previous and current surveys}

In this latest survey, $73.6 \%(187 / 254)$ of the responders answered 'Yes' to the question as to whether he/she thought that the Japanese government should restart its HPV vaccine recommendation (Table 2). This rate was significantly higher $(p=0.0027)$ than that in the previous study, which was $61.0 \%$ [7]. The rate of intending to recommend the HPV vaccine for teenagers also increased, from $65.2 \%$ to $70.1 \%$. Interestingly, in the present survey, 5 (16.1\%) of 31 doctors had their own teenaged daughters inoculated with HPV vaccine, even though the governmental recommendation was still suspended, whereas none of the doctors in the previous survey had done so. 
Table 1 Characteristics of the responders of the present survey

\begin{tabular}{|c|c|c|}
\hline & $n$ & Percent (\%) \\
\hline Gender & 256 & \\
\hline Male & 167 & 65.2 \\
\hline Female & 89 & 34.8 \\
\hline Age & 257 & \\
\hline Twenties & 6 & 2.3 \\
\hline Thirties & 58 & 22.6 \\
\hline Forties & 52 & 20.2 \\
\hline Fifties & 58 & 22.6 \\
\hline Sixties & 51 & 19.8 \\
\hline Seventies or older & 32 & 12.5 \\
\hline Working status & 256 & \\
\hline Employed as a doctor & 148 & 57.8 \\
\hline Owner of a clinic or hospital as a doctor & 91 & 35.5 \\
\hline Other & 17 & 6.7 \\
\hline Medical specialty ${ }^{\mathrm{a}}$ & 299 & \\
\hline General obstetrics/gynecology & 202 & 67.5 \\
\hline Obstetrics & 29 & 9.7 \\
\hline Gynecology & 37 & 12.4 \\
\hline Fertility & 14 & 4.7 \\
\hline Other & 17 & 5.7 \\
\hline Experienced a cervical cancer patient & 257 & \\
\hline Yes & 252 & 98.1 \\
\hline No & 5 & 1.9 \\
\hline Experienced the death of a cervical cancer patient & 257 & \\
\hline Yes & 226 & 87.9 \\
\hline No & 31 & 12.1 \\
\hline Have a child & 256 & \\
\hline Yes & 205 & 80.1 \\
\hline No & 51 & 19.9 \\
\hline Have a daughter who is less than 30 years old & 255 & \\
\hline Yes & 206 & 80.8 \\
\hline No & 49 & 19.2 \\
\hline
\end{tabular}

Response with no answer excluded

${ }^{a}$ Multiple answers permitted

\section{Survey of responses on opinions toward the WHO statement and the Sobue Report}

Physician recognition of the WHO statement [8] and the Sobue Report [9] was analyzed. A part of the Global Advisory Committee on Vaccine Safety's 'Statement on Safety of HPV Vaccines' released on 17 December 2015 and the Conclusion section of the Sobue Report were provided to the doctors (Fig. 1). Among the responders, 66.5\% were aware of the WHO statement, and $71.5 \%$ were aware of the Sobue epidemiological study (Table 3). Further, $72.7 \%$ of respondents agreed with the safety findings for the HPV vaccine and 84.3\% agreed on the effectiveness of HPV vaccine.

\section{Change of opinions toward the HPV vaccine after being provided with information regarding the WHO statement and the Sobue report}

As a baseline for the present study, 26.3\% (67/254) of the responding doctors answered 'No' or 'Unclear' to the question as to whether he/she thought that the Japanese government should restart its recommendation, and 29.9\% (76/254) of responders answered 'No' or 'Unclear' to the question as to whether he/she recommended HPV vaccine for teenagers. Among those, 12 of 67 doctors changed their answer to 'Yes' after looking at the WHO statement, and 5 of 67 doctors switched to 'Yes' after reading the Sobue Report (Table 4).

\section{Discussion}

The incidence rate of cervical cancer has been increasing steadily in Japan [2-4]. Unfortunately, the inoculation rate of HPV vaccine has catastrophically declined. Almost 4 years have passed since the announced recommendation suspension of HPV vaccine by the government, and the number of females who have had their first sexual encounters without the benefits of a protective HPV vaccination has been ever increasing during this period. We previously predicted that 2016 was the last chance we had to minimize the potential risk of HPV infection for Japanese girls by resuming the government's recommendation, and that high-infection-rate groups will be generated for every 1-year delay in resuming that recommendation $[10,11]$. Despite our warning, the government's recommendation remains suspended until May 2017.

We conducted three investigations, by using the Internet method, about the thoughts on HPV vaccine of mothers who had unvaccinated 12- to 16-year-old girls in Japan: at 9 months (March 2014), at 23 months (May 2015), and 32 months (February 2016) [12], after the announcement of
Table 2 Comparison of baseline opinions toward HPV vaccine between the previous and the present survey

\begin{tabular}{llll}
\hline & Previous survey $^{\mathrm{a}}$ & Present survey & $p$ value \\
\hline Government should restart its recommendation & $61.0 \%(161 / 264)$ & $73.6 \%(187 / 254)$ & 0.0027 \\
I recommend HPV vaccine for teenagers & $65.2 \%(172 / 264)$ & $70.1 \%(178 / 254)$ & 0.26 \\
I had my teenaged daughter inoculated & $0 \%(0 / 18)$ & $16.1 \%(5 / 31)$ & 0.14 \\
\hline
\end{tabular}

${ }^{\text {a }}$ The previous survey was conducted in 2014 [7] 
(a) Part of the WHO Global Advisory Committee on Vaccine Safety Statement on 'Safety of HPV vaccines' December 17, 2015 [8]

The GACVS has systematically investigated safety concerns raised about HPV vaccines and has issued several reports in this regard. To date, it has not found any safety issue that would alter its recommendations for the use of the vaccine. (snip)

The circumstances in Japan, where the occurrence of chronic pain and other symptoms in some vaccine recipients has led to suspension of the proactive recommendation for routine use of vaccine in the national immunization program, warrants additional comment. Review of clinical data by the national expert committee led to a conclusion that symptoms were not related to the vaccine, but it has not been possible to reach consensus to resume HPV vaccination. As a result, young women are being left vulnerable to HPV-related cancers that otherwise could be prevented. As GACVS has noted previously, policy decisions based on weak evidence, leading to lack of use of safe and effective vaccines, can result in real harm.

(b) Conclusion of the report of a Japanese nation-wide epidemiologic study [9]

The conclusion of a nation-wide epidemiologic study of adolescents in Japan regarding diverse symptoms, including pain and motor disability, conducted under MHLW financial support

1. There were adolescents who did not receive HPV vaccination who suffered from symptoms similar to those observed in HPV vaccinated girls.

2. This study could not evaluate the causal association of HPV vaccination on diverse symptoms observed in vaccinated girls.

Fig. 1 World Health Organization (WHO) statement and the Sobue Report shown in the present survey

Table 3 Survey answers regarding the WHO statement and the report of Japanese nation-wide epidemiological study (Sobue Report)

\begin{tabular}{lll}
\hline & Was aware & $\begin{array}{l}\text { Confirming } \\
\text { safety/effective- } \\
\text { ness }\end{array}$ \\
\hline WHO statement $^{\mathrm{a}}$ & $66.5 \%(169 / 254)$ & $72.7 \%(178 / 245)$ \\
$\begin{array}{l}\text { Report of epidemiologi- } \\
\text { cal study }\end{array}$ & $71.5 \%(178 / 249)$ & $84.3 \%(209 / 248)$ \\
\hline
\end{tabular}

${ }^{a}$ Global Advisory Committee on vaccine safety statement on 'Safety of HPV vaccines,' December 17, 2015 [8]

${ }^{\mathrm{b}}$ The conclusion of the report of the Japanese nation-wide epidemiological study [9]

Table 4 Change of opinions toward HPV vaccine after reading information provided regarding the WHO statement and the Sobue Report

Positive change with the information ${ }^{\mathrm{a}}$ provided

Government should restart recommendation

$12 / 67$

I recommend HPV vaccine for teenagers

$5 / 76$

${ }^{a}$ The number of the obstetricians and gynecologists whose answer was changed from 'No' or 'Unclear' in the baseline question to 'Yes' after being provided with the information of the WHO statement [8] and the report of the Japanese nation-wide epidemiological study [9] the suspension of the government recommendation. From our results, those mothers' intention for acceptance of HPV vaccination decreased with time (unpublished data).

Our first survey conducted in August 2014, 14 months after the announcement of the suspension of recommendation, on the opinions about the HPV vaccine of our obstetricians and gynecologists, revealed that most obstetricians and gynecologists were negatively influenced as the general population by media reports of so-called adverse events of the HPV vaccine and by the suspension of the governmental recommendation as well as the general public.

In this report, we present the results of a follow-up second survey, conducted on the same target doctors, on the same issues. During the last 3 years, the doctors' opinion toward HPV vaccine had changed to a positive direction. The percent of the doctors who thought that the Japanese government should restart recommendation significantly increased ( $p=0.0027$ ), and the percent of the doctors who recommend HPV vaccine for teenagers had also increased, however, not significantly, implying that strong recommendation of HPV vaccine for teenagers is still difficult under the suspension of the governmental recommendation. These results were not different in male and female doctors (data not shown).

In the previous survey there were no doctors who had inoculated their own teenaged daughters with the HPV vaccine. However, in the present study 5 of 31 doctors did inoculate with HPV vaccine. These positive results may give some positive impression to the general public regarding the safety of HPV vaccine. However, the WHO statement that referred to the circumstances in Japan and the report of the nation-wide epidemiological study (the Sobue Report), which appeared after our previous survey, had unquestionable positive impact on the intention of doctors. The information had a role in changing the opinion of the doctors who had previously been negative toward the HPV vaccine. Now the majority of the responders recognize that the HPV vaccine is safe and effective. These doctors' positive opinion will be a potential driving force for a new dissemination of the HPV vaccine, because a trusted medical provider's recommendation has been shown to be key to improving vaccine usage [6].

We previously reported that the final decision maker of adolescents' HPV vaccination status in Japan was usually their mother [5, 11]. Most mothers, with or without consulting their husbands, decided to inoculate their daughters without any consideration for their daughters' feelings. Even now mothers are hesitating to recommend inoculation with HPV vaccine to their daughters because of the suspension of the governmental recommendation. At first mothers required a restart of the recommendation, and next they want see girls of a similar age as their daughter get safely vaccinated before they will encourage their own daughter to get vaccinated [11]. Creating a significant and positive change in the 
opinions of obstetricians and gynecologists toward the HPV vaccine should make it easy for mothers in the general population to decide to accept HPV vaccination for their daughters. This improved doctor perception can be accomplished by detailed explanations of the usefulness of the vaccine, including the information from the WHO statement and the Sobue Report. The most recent change of their mother's perceptions of the risk and the benefits of HPV vaccine will be described in the near future.

The positive results in the present study should further assure the general public regarding the safety of the HPV vaccine. It may be time for the government to consider resuming recommendation of the vaccine.

Acknowledgements We thank Dr. Masumi Sawada for his constructive critique of the questionnaire; Ms. Kanako Sakiyama, Ms. Rie Teramoto, Ms. Natsumi Mizuno, Ms. Ayako Okamura, and Ms. Mami Morikawa for their support in conducting the survey, and Dr. G.S. Buzard for his constructive critique and editing of our manuscript.

\section{Compliance with ethical standards}

Conflict of interest This study was approved by our Institutional Review Board and Ethics Committee. Yutaka Ueda received a grant from the Japan Agency for Medical Research and Development (Grant Number 15ck0106103h0102) and has received lecture fees from GlaxoSmithKline/Japan Vaccine and received lecture fees, research funds, and consultation fees from Merck Sharp \& Dohme. Asami Yagi received a lecture fee from Merck Sharp \& Dohme. Tadashi Kimura received a lecture fee from GlaxoSmithKline/Japan Vaccine and research funds from Merck Sharp \& Dohme.

Open Access This article is distributed under the terms of the Creative Commons Attribution-Non Commercial 4.0 International License (http://creativecommons.org/licenses/by-nc/4.0/), which permits any noncommercial use, distribution, and reproduction in any medium, provided you give appropriate credit to the original author(s) and the source, provide a link to the Creative Commons license, and indicate if changes were made.

\section{References}

1. Japanese Ministry of Health, Labour and Welfare. Vital Statistics in Japan, tabulated by the Center for Cancer Control and Information Services, National Cancer Center, Japan. Available at: http:// ganjoho.jp/pro/statistics/en/table_download.html. Accessed Apr 3, 2017

2. Ueda Y et al (2015) Japan's failure to vaccinate girls against human papillomavirus. Am J Obstet Gynecol 212:405-406

3. Hanley SJ et al (2015) HPV vaccination crisis in Japan. Lancet 385:2571

4. Sekine $M$ et al (2016) Japanese crisis of HPV vaccination. Int J Pathol Clin Res IJPCR-2-039 2(2):1-3

5. Egawa-Takata et al (2015) Survey of Japanese mothers of daughters eligible for human papillomavirus vaccination on attitudes about media reports of adverse events and the suspension of governmental recommendation for vaccination. J Obstet Gynaecol Res 41:1965-1971

6. Bratic JS et al (2016) Update on barriers to human papillomavirus vaccination and effective strategies to promote vaccine acceptance. Curr Opin Pediatr 28:407-412

7. Egawa-Takata et al (2016) Human papillomavirus vaccination of the daughters of obstetricians and gynecologists in Japan. Int J Clin Oncol 21:53-58

8. Global Advisory Committee on vaccine safety statement on safety of HPV vaccines on 17 December 2015. Available at: http://www.who.int/vaccine_safety/committee/GACVS_HPV_ statement_17Dec2015.pdf\#search $=\% 27 \mathrm{Global}+$ Advisory+Co mmittee+on+Vaccine+safety+Statement+on+Safety+of+HPV +vaccines\%27. Accessed Apr 3, 2017

9. A joint meeting of the Vaccine Adverse Reactions Review Committee (VARRC). Available at: http://www.mhlw.go.jp/file/05Shingikai-10601000-Daijinkanboukouseikagakuka-Kouseikagakuka/0000147016.pdf. Accessed Apr 3, 2017

10. Tanaka $\mathrm{Y}$ et al (2016) Outcomes for girls without HPV vaccination in Japan. Lancet Oncol 17:868-869

11. Yagi A et al (2017) Realistic fear of cervical cancer risk in Japan depending on birth year. Hum Vaccin Immunother. https://doi. org/10.1080/21645515.2017.1292190

12. Yagi A et al (2016) Development of an efficient strategy to improve HPV immunization coverage in Japan. BMC Public Health 16:1013 